\title{
Théorie de la méthode d'Allen et ses conséquences pratiques pour la mesure des débits en conduite
}

PAR

\section{J. Guizerix}

Chef du Groupe

des Applications de la Radioactivité et des Traceurs en Hydrologie at du
La méthode d'Allen pour la mesure de débits en conduite est ancienne puisque c'est en 1923 qu'Allen la présente sous le titre "The salt velocity method of water measurement". (Réf. 1).

La technique de cette méthode consiste à injecter un traceur dans une section ( $\Sigma$ figure 1) d'une conduite, et à relever dans deux sections $\Sigma_{1}$ et $\Sigma_{2}$ situées en aval les variations de concentration, $C_{1}(t)$ et $C_{2}(t)$ qui traduisent le passage du traceur dans ces sections. Dans les expériences d'Allen le traceur était un sel $(\mathrm{ClNa})$ dont la concentration était déterminée par mesure de conductibilité. La figure 1 est relative à une technique classique dans laquelle le traceur est radio-actif ; les concentrations sont mesurées à l'aide de détecteurs disposés à l'extérieur de la conduite.

Une condition généralement imposée à l'application de cette méthode est que la conduite soit rectiligne entre $\Sigma_{1}$ et $\Sigma_{2}$ et que sa section $S$ soit constante. Pour calculer le débit on applique la relation

$$
Q=\frac{S x}{t}, x=\text { distance entre } \Sigma_{1} \text { et } \Sigma_{2}
$$

Suivant les auteurs $t$ représente la différence des abscisses des sommets de $C_{1}(t)$ et $C_{2}(t)$, la différence des abscisses des médianes, des centres de gravité ou de "points homologues" comme le milieu du segment déterminé par $C_{1}(t)$ et $C_{2}(t)$ sur une parallèle à l'axe des abscisses, au tiers de l'ordonnée maximale de chaque fonction (Réf. 2 et 3 ).

A l'origine et pour beaucoup d'expérimentateurs, cette méthode serait basée sur la mesure de vitesse d'un certain "piston" représenté par le nuage de traceur. La relation (1) peut en effet s'écrire $Q=S \frac{x}{t}=S \times$ vitesse ce qui justifie l'appellation anglo-saxonne de la méthode : "pulse velocity method". Or le "piston" se déforme au cours de son déplacement, d'où la difficulté de définir $t$.

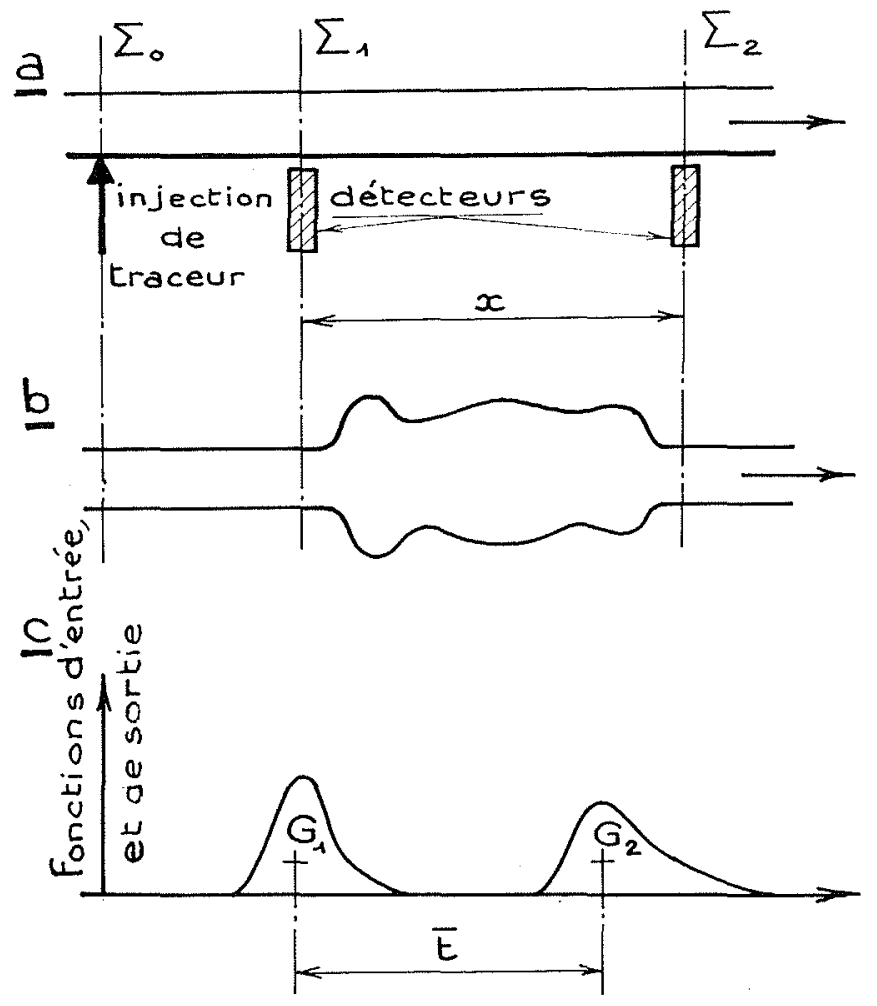

$$
\Phi=\frac{V}{E} \quad \bar{E}=\int_{-\infty}^{+\infty} t \cdot h_{\Sigma_{1} \Sigma_{2}}(t) d t
$$

1/Méthode d'Allen 


\section{La méthode d'Allen n'est pas fondée sur une détermination de la vitesse moyenne}

Les adeptes du choix de la différence des abscisses des centres de gravité de $C_{1}(t)$ et de $C_{2}(t)$ écrivent :

$$
Q=\frac{V}{t}=\frac{S x}{t}=S \bar{v}
$$

$\bar{v}$ représentant une vitesse moyenne.

Cette écriture est inexacte sur le plan mathématique, et n'est pas fondée sur le plan de la physique.

Sur le plan mathématique :

$$
\bar{v}=\left(\frac{\bar{x}}{t}\right) \neq \frac{x}{\bar{t}}
$$

La moyenne des vitesses des molécules d'eau entre $\Sigma$ et $\Sigma$, n'est pas en général égale au quotient de la distance par la moyenne des temps de transit.

Si $h_{\Sigma_{1} \Sigma_{2}}(t)$ dt représente la distribution des temps de séjour de l'eau dans le tronçon défini par $\Sigma_{1}$ et $\Sigma_{2}$ rappelons que :

$$
\bar{t}=\int_{-\infty}^{+\infty} t \cdot h_{\Sigma_{1} \underline{-}_{2}}(t) d t
$$

et

$$
\bar{v}=x \int_{-\infty}^{+\infty} \frac{1}{t} h \underset{\substack{* \\ \Sigma_{1}=2}}{ }(t) d t
$$

(Réf. 4 et le mémoire "Méthodologie d'étude par traceur des transferts de masse" de la présente réunion).

Sur le plan de la physique, à la notion de débit correspond une notion de flux. Un débit est égal au produit d'une aire par une vitesse, cette dernière (vitesse moyenne dans le temps et dans l'espace de la section) est définie dans une section. On n'est pas autorisé, en général, à multiplier une aire par la vitesse moyenne définie entre deux sections pour obtenir un débit.

La méthode d'Allen repose, en fait, sur l'application d'une relation utilisée, par exemple, par les spécialistes des transferts de masse dans le domaine du génie chimique :

$$
Q=\frac{V}{\bar{t}}
$$

où $V$ représente le volume d'un système parcouru par un écoulement de débit $Q$, et $\bar{t}$ est le premier moment de la distribution des temps de séjour, (cf. définition (3) ci-dessus). On en trouvera une démonstration dans les mémoires des références [5] et [6]. Nous représentons ci-dessous une démonstration que nous avons exposée dans le texte de la référence [4].

\subsection{ETABLISSEMENT DE LA RELATION $Q=\frac{V}{\bar{t}}$}

Soit $h(t)$ la distribution des temps de séjour d'un fluide incompressible dans un système limité par une section d'entrée et une section de sortie (figure 2), l'écoulement se faisant à débit constant $Q$. Nous supposerons ce système fermé à la diffusion à l'amont et à l'aval.

Définissons la réponse indicielle $j(t)$ du système par :

$$
j(t)=\int_{0}^{t} h(u) d u .
$$

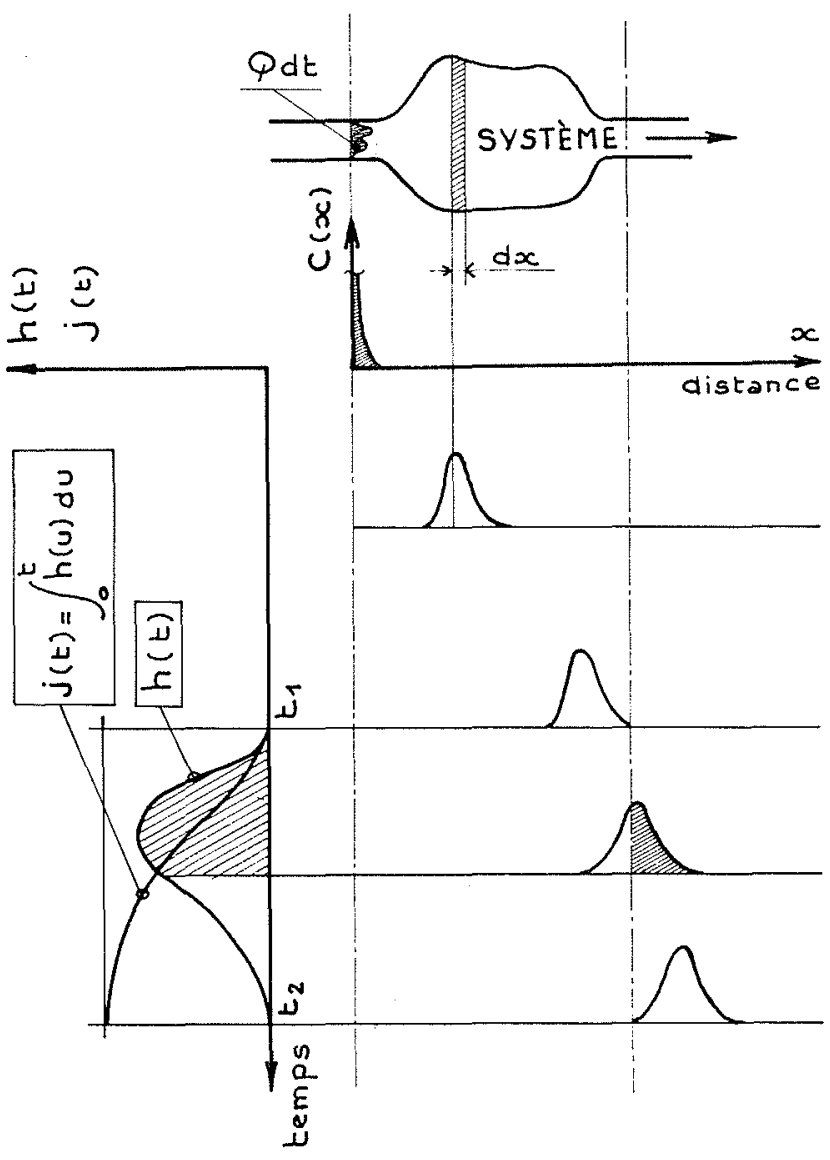

$2 /$ Correspondance entre $C(x)$ et $h(t)$

Nous nous intéressons à l'évolution d'un échantillon de matière de volume $Q d t$ défini par l'ensemble des molécules qui ont pénétré dans le système entre $t_{\mathrm{o}}$ et $t_{\mathrm{o}}+i t$

La première molécule qui sort du système définit une date $t_{1}$, et la dernière qui en sort une date $t_{2}, t_{0}$ est pris comme origine des temps.

La fraction de l'échantillon déjà ressortie à une date $t$ est :

$$
\int_{0}^{t}[Q d t h(u)] d u=Q j(t) d t
$$

Ecrivons que :

(volume $V$ du système $)=($ volume du fluide entré entre $t=0$ et $\left.t_{2}\right)$ (fraction de ce volume ressortie avant $t_{2}$ )

$$
V=Q t_{2}-Q \int_{0}^{t_{2}} j(t) d t
$$

d'oủ la relation

$$
Q=\frac{V}{t_{2}-\int_{0}^{t_{2}} j(t) d t}
$$

que l'on peut mettre sous la forme :

$$
Q=\frac{V}{t_{1}+\int_{t_{1}}^{t_{2}}[1-j(t)] d t}
$$

Ces relations sont identiques à la relation $Q=\frac{V}{\bar{t}}$; montrons en effet que :

$$
t_{2}-\int_{0}^{t_{2}} j(t) d t \equiv \bar{t}
$$


ou que

$$
\int_{0}^{t_{2}} j(t) d t+\bar{t}=t_{2}
$$

Ecrivons pour cela la relation générale $(F \varphi)^{\prime}=f^{\prime} \varphi+f \varphi^{\prime}$ avec

$$
\begin{aligned}
& f=t \\
& \varphi=\int_{0}^{t} h(t) d t
\end{aligned}
$$

sous sa forme intégrale :

$$
\begin{aligned}
& \int_{0}^{t_{2}}\left[\int_{0}^{t} h(u) d u\right] d t+\int_{0}^{t_{2}} t h(t) d t= \\
& \qquad\left|t \int_{0}^{t} h(t) d t\right|_{0}^{t_{2}}=t_{2},
\end{aligned}
$$

ce qu'il fallait démontrer.

Reportons-nous à la figure la ; nous pouvons considérer trois systèmes définis par $\Sigma_{0}, \Sigma_{1}$ et $\Sigma_{2}$

$\left(\Sigma_{0} \Sigma_{1}\right),\left(\Sigma_{1} \Sigma_{2}\right)$ en série, et un système global $\left(\Sigma_{0} \Sigma_{2}\right)$

Si $\bar{t}_{1}, \bar{t}_{1}, \bar{t}_{2}$ représentent respectivement les premiers moments des distributions des temps de séjour des systèmes, $\left(\Sigma_{0} \Sigma_{1}\right),\left(\Sigma_{1} \Sigma_{2}\right),\left(\Sigma_{0} \Sigma_{2}\right)$ on montre que :

$$
\bar{t}=\bar{t}_{2}-\bar{t}_{1}
$$

Notons que les fonctions $C_{i}(t)$ et $h_{i}(t)$ ont des centres de gravité qui ont une même abscisse. On obtient donc $\bar{t}$ par la différence des abscisses des centres de gravité des fonctions $C_{2}(t)$ et $C_{1}(t)$. (figure $1 \mathrm{c}$ ).

\subsection{CONSEQUENCE PRATIQUE}

Pour obtenir le débit d'un écoulement de matière par la méthode d'Allen, il faut donc déterminer d'une part le premier moment de la distribution des temps de séjour et d'autre part le volume de la conduite entre les deux sections de mesure. Il n'est pas imposé que la conduite soit rectiligne et de diamètre constant entre ces sections comme cela était requis dans la présentation classique de cette méthode. (figure $1 \mathrm{~b}$ ).

\section{Conditions d'application de la méthode d'Allen}

La méthode d'Allen est applicable si certaines conditions sont respectées. Ces conditions sont imposées d'une part par la méthode et d'autre part par la technique.

\subsection{CONDITIONS IMPOSEES PAR LA METHODE}

Ce sont :

1) Le fluide sur lequel porte la mesure est un fluide incompressible.

2) Le débit est constant.

3) La condition de bon mélange

Soit $n(P, t)$ le nombre de particules de traceur en un "point" $P$, à l'instant $t$. (Une bonne approximation de cette fonction est $C_{i}(t)$, la formation concentration-temps en un "point" repéré par $i$ ). Le nombre de particules de traceur dans un volume $d V$ entre $t$ et $t+d t$ est :

$$
n(P, t) d V d t
$$

Le bon mélange s'exprime par :

$\int_{0}^{\infty} n(P, t) d t=R=$ constante (indépendante de $P$ )

Soient : $u(t)$ le flux de traceur dans $\Sigma_{1}$ (figure 1) $y(t)$ le flux de traceur dans $\Sigma_{2}$

$M(t)$ le nombre de particules de traceur présentes à l'instant $t$ dans le volume $V$.

On a :

$$
u(t)=\int_{\Sigma_{1}} n(P, t) \vec{V}(P) \vec{n} d s
$$

où $\vec{V}(P)=$ vecteur vitesse au point $P$ et $\vec{n}$ la normale à $d s$

$$
\begin{gathered}
y(t)=\int_{\Sigma_{2}} n(P, t) \vec{V}(P) \vec{n} d s \\
M(t)=\int_{\nu} n(P, t) d V
\end{gathered}
$$

La relation de bilan s'écrit :

$$
\frac{d M}{d t}=u(t)-y(t)
$$

Multiplions les deux membres par $t$ et intégrons de 0 à l'infini :

$$
\int_{v} \int_{0}^{\infty} t \frac{\partial n(P, t)}{\partial t} d t=\int_{0}^{\infty} t u(t) d t-\int_{0}^{\infty} t y(t) d t
$$

Une intégration par parties donne :

$$
\int_{0}^{\infty} t \frac{\partial n(P, t)}{\partial t} d t|t \cdot n(P, t)|_{0}^{\infty}-\int_{0}^{\infty} n(P, t) d t
$$

Si la condition de bon mélange est respectée l'expression au second membre est égale à $-\mathrm{R}$. On a ainsi

$$
-R V=\int_{0}^{\infty} t u(t) d t-\int_{0}^{\infty} t y(t) d t
$$

Soient $\bar{t}_{1}$ et $\bar{t}_{2}$ les premiers moments des flux entrant et sortant :

$$
\bar{t}_{1}=\frac{\int_{0}^{\infty} t \cdot u(t) d t}{\int_{0}^{\infty} u(t) d t} ; \bar{t}_{2}=\frac{\int_{0}^{\infty} t y(t) d t}{\int_{0}^{\infty} y(t) d t}
$$

Le flux de traceur étant conservatif

$$
\int_{0}^{\infty} u(t) d t=\int_{0}^{\infty} y(t) d t
$$

d'où :

$$
\begin{aligned}
& \int_{\Sigma_{1}} \int_{0}^{\infty} n(P, t) \vec{V}(P) \cdot \vec{n} d s d t= \\
& \quad \int_{\Sigma_{2}} \int_{0}^{\infty} n(P, t) \vec{V}(P) \vec{n} d s d t
\end{aligned}
$$

La condition de bon mélange étant respectée :

$$
\int_{0}^{\infty} u(t) d t=\int_{0}^{\infty} y(t) d t=R \int_{0}^{\infty} \vec{V}(P) \vec{n} d s=K Q
$$

d'où :

$$
\frac{V}{Q}=\bar{t}_{2}-\bar{t}_{1}
$$

Cette relation a été établie en ne supposant que le respect de la condition de bon mélange.

Remarque. Cette nouvelle démonstration de la relation $Q=\frac{V}{\bar{t}_{2}-\bar{t}_{1}}$ ne fait pas double emploi avec celle qui a été pré- 
sentée au paragraphe 1.1. La première avait trait en effet à un système linéaire scalaire invariant et la seconde au cas réel d'un système linéaire vectoriel invariant (cf. mémoire "Méthodologie d'étude par traceur des transferts de masses").

Nous avons montré que la condition de bon mélange était une condition nécessaire pour l'application de la méthode d'Allen. Existe-t-il une section dans laquelle le bon mélange est obtenu? Nous examinons maintenant cette question.

Existence d'une section pour laquelle le bon mélange est réalisé

On peut trouver en aval de $\Sigma_{0}$ une section $\Sigma_{1}$ pour laquelle

$$
\int_{-\infty}^{+\infty} C_{i}(t) d t=\mu \pm \epsilon
$$

où $\mu$ est une constante et $\epsilon$ peut avoir une valeur aussi petite qu'on le désire. On dit que le bon mélange est réalisé à $100 \epsilon / \mu \%$ près.

Soient en aval de $\Sigma_{0}$ (section d'injection) des sections $S_{1}$, $S_{2}, \ldots$ équidistantes. Nous nous intéressons au mouvement d'une molécule d'eau en aval de $\Sigma_{0}$.

Cette molécule traverse chaque élément $d s_{i}$ de $\Sigma_{0}$ avec des probabilités différentes que nous représentons par un vecteur:

$$
\vec{\gamma}_{0}=\left(P_{1}^{\circ}, P_{2}^{\circ}, \ldots, P_{m}^{\circ}\right)
$$

Nous représentons également par un vecteur l'ensemble des probabilités de passage de cette molécule dans un élément d'une section $S_{\sigma}$

$$
\vec{\gamma}_{\sigma}=\left|P_{i}^{\sigma}\right| \quad i=1,2, \ldots, m
$$

Considérons deux sections consécutives $S_{K}$ et $S_{K+1}$ et repèrons par $i$ et $j$ les éléments de surface homologues, les deux sections étant supposées identiques.

Représentons par une matrice l'ensemble des probabilités de passage de la molécule d'un élément $d s_{i}$ de $S_{K}$ à un élément $d s_{j}$ de $S_{K+1}$.

$$
\Pi=\left|\alpha_{i, j}^{K, K+1}\right| \quad \begin{aligned}
i & =1,2, \ldots, m \\
j & =1,2, \ldots, m
\end{aligned}
$$

Supposons que cette matrice soit indépendante de $K$; on traduit ainsi la stationnarité de l'écoulement.

Nous pouvons alors considérer que le mouvement de la molécule correspondante se fait selon un processus de Markov homogène du premier ordre

On a :

$$
\vec{\gamma}_{\sigma}=\vec{\gamma}_{0} \Pi^{\sigma}
$$

on démontre que si l'on est dans le cas "positivement régulier" $\Pi^{\sigma}$ tend, lorsque $\sigma$ croît, vers une matrice dont toutes les lignes sont identiques

$$
\vec{\gamma}_{\infty}=\left(P_{1}^{\circ}, P_{2}^{\circ}, \ldots, P_{m}^{\circ}\right) \times\left|\begin{array}{c}
\alpha_{1} \alpha_{2} \ldots \ldots . \alpha_{m} \\
\alpha_{1} \alpha_{2} \ldots \ldots . \alpha_{m} \\
\ldots \ldots \ldots \ldots . \\
\alpha_{1} \alpha_{2} \ldots \ldots . \alpha_{m}
\end{array}\right|
$$

d'où

$$
\vec{\gamma}_{\infty}=\left(\alpha_{1}, \alpha_{2}, \ldots, \alpha_{m}\right)
$$

On montre que cette convergence vers un "régime permanent" est de caractère exponentiel ; elle est donc rapide.
Par conséquent on doit pouvoir trouver en aval de $\Sigma_{0}$ une section $S_{p}$ pour laquelle les probabilités de passage

$$
\begin{aligned}
& P_{1}^{p} \cong \alpha_{1} \\
& P_{2}^{p} \cong \alpha_{2} \\
& \cdots \cdots \\
& P_{m}^{p} \cong \alpha_{m}
\end{aligned}
$$

sont indépendantes des conditions initiales.

$\mathrm{Si}$ au lieu d'une molécule, on considère $N$ molécules en évolution dans le système, les fréquences relatives de passage de ces molécules dans les éléments de surface de $S_{p}$ convergent (pour $N \rightarrow \infty$ ) en probabilité vers les probabilités de passage $P_{\mathrm{I}}^{p}, P_{2}^{p}, \ldots$

Soit $q_{i}$ le débit associé à l'élément de surface $d S_{i}$ on a done :

$$
P_{i}^{p}=\frac{q_{i}}{Q}
$$

La même analyse peut être faite pour décrire l'évolution des molécules d'un traceur en aval de $\Sigma_{0}$.

Nous dirons que le traceur est un bon traceur si sa matrice de passage est identique à celle de l'eau.

Si la masse de traceur injectée dans $\Sigma_{\mathrm{o}}$ est $A$ et si $C_{i}(t)$ est sa concentration dans l'élément de surface $d s_{i}$ de $S_{p}$ on a :

$$
P_{i}^{p}=\frac{q_{i} \int_{-\infty}^{+\infty} C_{i}(t) d t}{A}
$$

(8) et (9) permettent d'écrire :

$$
\int_{-\infty}^{+\infty} C_{i}(t) d t=\frac{A}{Q}=\text { constante }
$$

\subsection{CONDITION IMPOSEE PAR LA TECHNIQUE : IDENTITE DES REPONSES IMPULSIONNELLES}

Nous avons vu que la méthode d'Allen implique que l'on connaisse les fonctions d'entrée et de sortie du traceur en $\Sigma_{1}$ et $\Sigma_{2}$

Ces fonctions sont :

$$
\begin{aligned}
& u(t)=\Sigma q_{i} C_{i}(t) \\
& y(t)=\Sigma q_{j} C_{i}(t)
\end{aligned}
$$

Il faut donc, théoriquement, en un certain nombre de points mesurer $C_{i}(t)$ et $C_{j}(t)$ ainsi que les débits correspondants $q_{i}$ et $q_{j}$.

Il y a là quelque paradoxe car la connaissance des $q_{i}$ ou des $q_{j}$ dispenserait d'appliquer la méthode d'Allen. Ceci ne manque pas de nous faire penser à une tautologie.

Certains proposent de faire une mesure de concentration (par conductimétrie) en un ou plusieurs points bien définis. Cette procédure ne peut, à notre sens, avoir qu'une justification expérimentale.

La mesure d'un traceur radioactif à l'aide d'un détecteur disposé à l'extérieur pose un problème difficile. On sait que le détecteur en effet ne "voit" pas avec la même sensibilité les différentes parties de son volume sensible. On a donc affaire à des fonctions $C_{i}(t)$ déjà pondérées selon un certain mode par le détecteur et qu'il faudrait, de plus, pondérer par les débits. 
Cependant d'excellentes mesures de débits sont couramment réalisées avec des traceurs radioactifs par la méthode d'Allen. Nous en saisirons plus loin la raison.

Si les réponses impulsionnelles définies entre le point d'injection et chaque point de $\Sigma_{1}$ et chaque point de $\Sigma_{2}$ étaient identiques, il en résulterait que les différentes fonctions $C_{i}(t)$ seraient affines (identiques à un facteur constant près) ; de même pour les fonctions $C_{j}(t)$.

L'abscisse de leur centre de gravité serait la même et serait identique à celle du centre de gravité, respectivement, des fonctions

$$
\Sigma_{i} q_{i} C_{i}(t) \text { et } \Sigma_{j} q_{j} C_{j}(t)
$$

quelles que soient les valeurs des $q_{i}$ ou $q_{j}$. On obtiendrait alors avec une sonde nucléaire une valeur correcte de $t_{2}-t_{1}$. Si l'on déterminait en un seul point $C(t)$ dans $\Sigma_{\mathrm{i}}$ et $\Sigma_{2}$ par conductimétrie, la différence des abscisses des centres de gravité fournirait une valeur correcte de $\bar{t}$.

Nous allons montrer que l'on peut trouver une section pour laquelle cette propriété d'identité des réponses impulsionnelles existe.

Existence d'une section pour laquelle la condition d'identité des réponses impulsionnelles est satisfaite.

Soient $\Sigma_{0}$ la section d'injection et $\Sigma_{d}$ la section recherchée.

Nous supposerons que le traceur est injecté selon une impulsion de Dirac et que l'écoulement peut être représenté par un ensemble de systèmes élémentaires, associés en série et en parallèle comme le représente la figure 3 . Les systèmes, sur

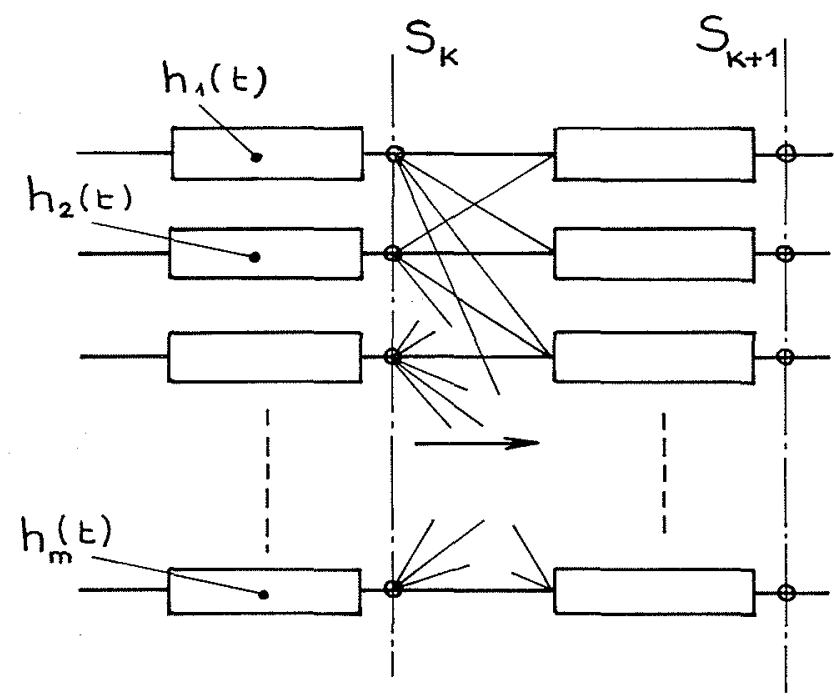

3/ Modète du processus de Markov

chaque ligne, ont même distribution des temps de séjour $h_{i}(t)$, et nous supposerons que le temps de passage d'une ligne à une autre est négligeable. Supposons enfin que la probabilité pour une molécule de se trouver aux différents noeuds peut être analysée selon un processus de Markov, homogène dans le temps et dans "le cas régulier". Ces suites de Markov ont la propriéé d'ergodicité : les moyennes statistiques convergent vers les probabilités $\alpha_{i}$ lorsque $s$ (qui définit "l'étage") tend vers l'infini. Rappelons que la moyenne statistique d'un évènement repéré par l'indice $i$ est le rapport du nombre $z_{i}$ de ses réalisations au nombre $s$ de fois où il aurait pu se produire :

On a :

$$
\frac{Z_{i}}{s} \frac{\text { Prob. }}{s \rightarrow \infty} \alpha_{i}
$$

Une molécule arrivant à un noeud quelconque d'une section $\Sigma_{d}$ aura séjourné dans les lignes $1,2, \ldots m$ avec des fréquences $\alpha_{1}, \alpha_{2}, \ldots, \alpha_{m}$. La réponse impulsionnelle $r(t)$ relative à chaque noeud sera identique et aura pour valeur :

$$
r(t)=h_{1}^{* \alpha_{1} d}(t) * h_{2}^{* \alpha_{2} d}(t) * \ldots h_{d}^{* \alpha_{d} d}(t)
$$

L'exposant $* \alpha_{i} d$ indique qu'il faut convoluer $\alpha_{i} d$ fois la fonction par elle même.

Cette propriété d'identité des réponses impulsionnelles a une très grande importance pratique. Elle évite de relever en de nombreux points les fonctions concentration-temps et la pondération de ces fonctions par les débits. A la limite une seule fonction concentration temps est nécessaire.

En pratique on en relève souvent au moins deux afin de vérifier que les réponses impulsionnelles sont identiques.

On montre (réf. 4) que cette propriété peut être étendue à un écoulement non confiné. Nous avons pu en faire de nombreuses vérifications expérimentales tant sur des écoulements à surface libre que sur des écoulements souterrains.

La section $\Sigma_{d}$ dans laquelle est obtenue l'identité des réponses impulsionnelles avec une bonne approximation est d'autant plus rapprochée de $\Sigma_{0}$ que l'écoulement est turbulent. On admet qu'un nombre de Reynolds au moins de 7000 à 8000 autorise l'application de la méthode d'Allen telle que nous l'avons décrite.

\section{Remarque 1 : influence du volume sensible de mesure}

Nous avons vu au paragraphe 1.1 que les fonctions concentration-temps relevées dans les sections $\Sigma_{1}$ et $\Sigma_{2}$ permettaient de calculer les premiers moments des distributions des temps de séjour des systèmes $\left(\Sigma_{0} \Sigma_{1}\right),\left(\Sigma_{0} \Sigma_{2}\right),\left(\Sigma_{1}\right.$ $\Sigma_{2}$ ). Ces fonctions concentration-temps $C_{i}(t)$ représentent un élément des flux massiques $q_{i} C_{i}(t)$ qui ont été pris en compte dans notre analyse.

Or un flux massique est défini au travers d'une surface, et une mesure de concentration intéresse toujours un volume. Il en résulte que $q_{i} C_{i}(t)$ ne peut être qu'une approximation du flux.

Le volume de mesure est lui même un système, pour lequel on peut définir un flux d'entrée $\varphi_{e}(t)$, un flux de sortie $\varphi_{s}(t)$, et une réponse impulsionnelle $h_{m}(t)$. Le flux que nous désirons est $\varphi_{\mathrm{e}}(t)$. Le signal de l'appareil de mesure $S(t)$ est égal à un facteur d'étalonnage près à la concentration $C_{i}(t)$ du traceur dans le volume de mesure.

Nous avons :

$$
\varphi_{s}(t)=\varphi_{e}(t) * h_{m}(t)
$$

et

$$
C_{i}(t)=K s(t)=\int_{0}^{t} \varphi_{e}(t) d t-\int_{0}^{t} \varphi_{s}(t) d t
$$

Cette relation permet d'apprécier l'approximation

$$
\varphi_{e}(t) \simeq q_{i} C_{i}(t)
$$

Cette remarque était de nature générale. Dans l'application de la méthode d'Allen l'existence des volumes de mesure n'a théoriquement pas d'importance à condition qu'ils soient identiques dans chaque section.

En effet si $t_{m}$ représente le premier moment de $h_{m}(t)$, on a :

$$
\left(t_{2}+t_{m}\right)-\left(t_{1}+t_{m}\right)=\bar{t}
$$


Remarque 2 : cas des régimes laminaires et intermédiaires laminaires - turbulents

Le problème d'application de la méthode d'Allen en régime laminaire ne parait pas très bien résolu aujourd'hui et l'on doit considérer que cette question est encore en développement

Les études de Felder et Gardner (réf. 7 et 8 ) nous laissent penser qu'une solution représentant une bonne approximation pourrait être assez rapidement trouvée.

Par contre, il n'existe pas, à notre connaissance, d'analyses qui permettent d'utiliser avec sureté la méthode d'Allen dans les régimes intermédiaires laminaire - turbulent $(2000<$ Reynolds $<7000$ )

\section{Références}

[1] ALLEN (C.M.) et TAYLOR (E.A.) - The solt velocity method of water measurement - Annual Meeting of the Am. Soc. of Mec. Engin. New-York 3-6 Dec. 1923.
[2] CLAYTON (C.G.) et al. - The accuracy and precision of liquid flow measurement by radioactives isotopes. Radioisotope Tracers in Industry and Geophysics (C.R. Colloque Prague 1966), AIEA Vienne $1967,563$.

[3] ALQUiER (M.) et al - La notion de bon mélange dans l'emploi des traceurs. Isotope Hydrology 1970 (C.R. Colloque Vienne 1970) Vienne 1970 AIEA, 441.

[4] GUizERiX(J.) et al. - Analyse des informations fournies par les traceurs naturels ou artificiels dans l'étude des systèmes aquifères en hydrogéologie. Isotope Technique in groundwater hydrology 1974 (C.R. Colloque Vienne 1974) AIEA, Vienne 1974, 2, 377.

[5] DANCKWERTS (P.V.) - - Local residence -.. times in continuous flow systems. Chem. Eng. Sci. 1958, 9, 78.

[6] SPALDING (D.G.) - A note on mean residence - times in steady flow of arbitrary complexity. Chem. Eng. Sci. 1958, 9, 74.

[7] Felder (R.M.) et Gardner (R.P.) - Trans. Am. Nucl. Soc. $15,172,1972$.

[8] GARDNER et al. - Tracer concentration Responses and Moments for Measurements of Laminar Flow in Circular Tubes. Int. J. of Appl. Rad and Isot. 1973, 24, 253.

\section{Discussion}

Président : M. Roger Roche

M. Le Président remercie M. GUIZER IX pour son original exposé et ouvre la discussion.

Daus le cas des régimes laminaires, estime M. AlquiER (ENSEEIHT. Toulouse), il n'y a pas de difficultés particulières d'un point de vue théorique à appliquer la méthode d'ALLEN puisque la seule hypothèse faite pour l'établissement de la relation $Q=\frac{V}{t}$ est que le traceur soit un "bon traceur" et que la condition de "bon mélange" soit satisfaite En pratique, il peut être difficile de réalier cette condition, mais si les conditions d'injection permettent d'y satisfaire, il n'y a aucune raison de proscrire l'emploi de la méthode de la mesure du temps de transit ou méthode d'ALLEN.

Assurément, répond M. GUIZERIX, la relation générale est toujours valable. La difficulté majeure est que, dans le cas des écoulements laminaires, on ne sait pas mesurer un flux.

En l'absence d'autre intervention, M. Le Président donne la parole à M. L. Roche pour l'exposé de sa communication. 